\title{
The Buffer Zone of the Quasi-Biennial Oscillation $\mathscr{A}$
}

\author{
AARON MATCH \\ Program in Atmospheric and Oceanic Sciences, Princeton University, Princeton, New Jersey \\ STEPHAN FUEGLISTALER \\ Department of Geosciences, and Program in Atmospheric and Oceanic Sciences, Princeton University, \\ Princeton, New Jersey
}

(Manuscript received 30 May 2019, in final form 16 August 2019)

\begin{abstract}
The quasi-biennial oscillation $(\mathrm{QBO})$ is a descending pattern of winds in the stratosphere that vanishes near the top of the tropical tropopause layer, even though the vertically propagating waves that drive the QBO are thought to originate in the troposphere several kilometers below. The region where there is low QBO power despite sufficient vertically propagating wave activity to drive a QBO is known as the buffer zone. Classical one-dimensional models of the QBO are ill suited to represent buffer zone dynamics because they enforce the attenuation of the QBO via a zero-wind lower boundary condition. The formation of the buffer zone is investigated by analyzing momentum budgets in the reanalyses MERRA-2 and ERA-Interim. The buffer zone must be formed by weak wave-driven acceleration and/or cancellation of the wave-driven acceleration. This paper shows that in MERRA-2 weak wave-driven acceleration is insufficient to form the buffer zone, so cancellation of the wave-driven acceleration must play a role. The cancellation results from damping of angular momentum anomalies, primarily due to horizontal mean and horizontal eddy momentum flux divergence, with secondary contributions from the Coriolis torque and vertical mean momentum flux divergence. The importance of the damping terms highlights the role of the buffer zone as the mediator of angular momentum exchange between the QBO domain and the far field. Some far-field angular momentum anomalies reach the solid Earth, leading to the well-documented lagged correlation between the QBO and the length of day.
\end{abstract}

\section{Introduction}

The quasi-biennial oscillation $(\mathrm{QBO})$ is a descending pattern of easterly and westerly winds in the tropical stratosphere with a period of approximately 28 months. The QBO was discovered in 1960 (Reed et al. 1961; Ebdon and Veryard 1961). Although its downward propagation initially defied explanation, a theory for the QBO was developed in the late 1960s based on wavemean flow interactions (Lindzen and Holton 1968). Soon after, the theory was distilled into a one-dimensional model (Holton and Lindzen 1972), which still undergirds modern understanding of the downward propagation of QBO shear zones (Plumb 1977).

\footnotetext{
T) Supplemental information related to this paper is available at the Journals Online website: https://doi.org/10.1175/JAS-D-190151.s1.

Corresponding author: Aaron Match, amatch@princeton.edu
}

Key to the QBO mechanism is the interaction between vertically propagating waves and the mean flow. An obvious question to ask is whether the lower boundary of the QBO is given by the source level of the vertically propagating waves. Figure 1 shows that in terms of either zonal-mean zonal wind or axial relative angular momentum, QBO power (i.e., average spectral power of the zonal-mean zonal wind at QBO frequencies) vanishes as the wind anomalies approach the tropical tropopause. The exact source level of the waves remains an open question, but is generally thought to be in the troposphere, substantially below the vanishing level. Thus, observations suggest that the bottom of the QBO and the wave source are separated by a buffer zone (terminology from Saravanan 1990).

The buffer zone influences climate in at least two important ways. First, the upper troposphere-lower stratosphere region is likely where interactions between the mean fields of the QBO and the tropical troposphere occur, such as the proposed connection 

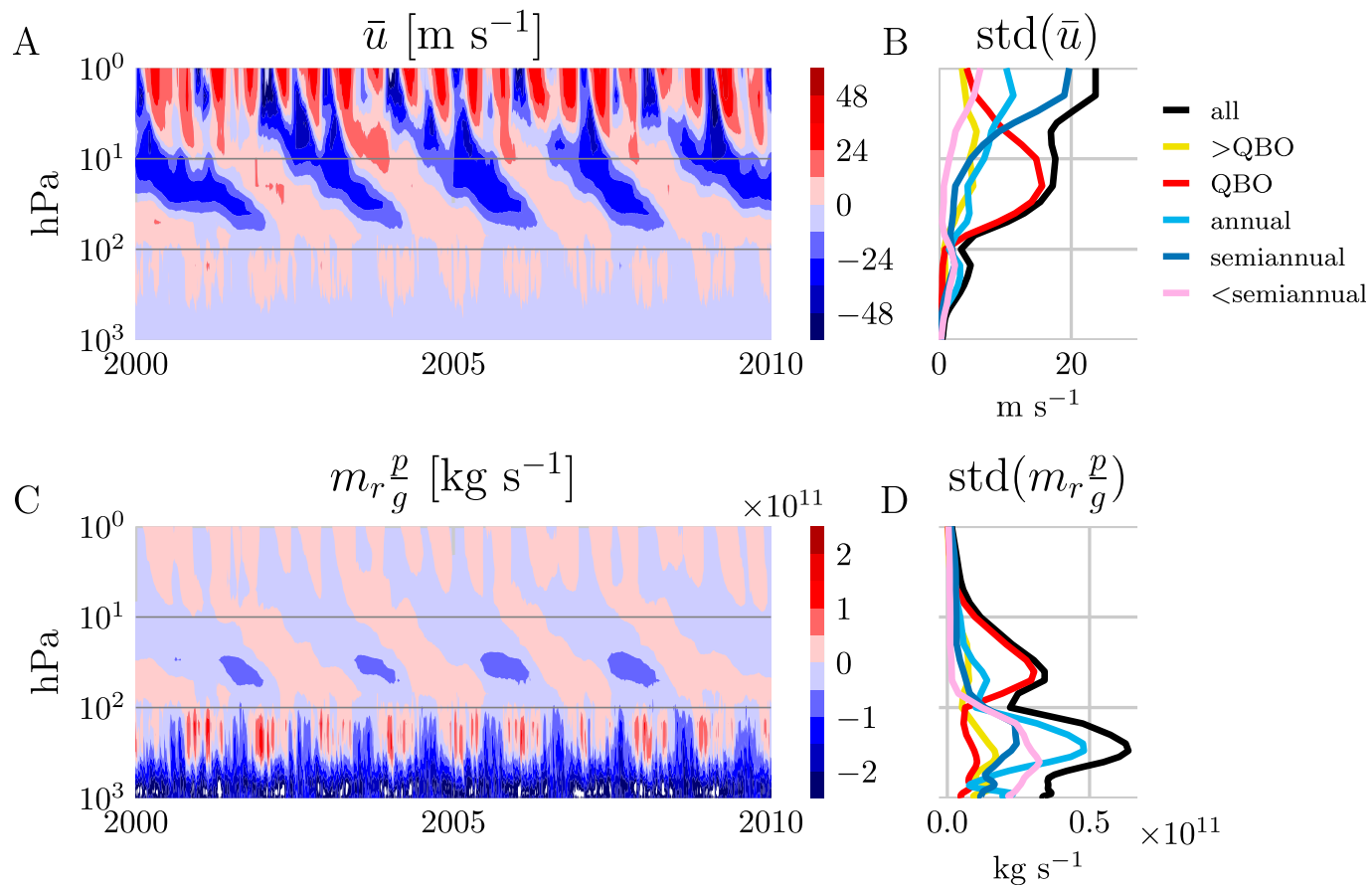

FIG. 1. Climatology of zonal wind and relative angular momentum in the deep tropics. (a) Zonal wind. (b) Standard deviation of zonal wind decomposed into spectral bands. (c) Relative angular momentum $m_{r}$ multiplied by log-pressure density (i.e., pressure divided by gravitational acceleration). (d) Standard deviation of relative angular momentum multiplied by log-pressure density decomposed into spectral bands. Data are from MERRA-2 and averaged in longitude and from $10^{\circ} \mathrm{S}$ to $10^{\circ} \mathrm{N}$. Standard deviations are for the period January 1980-November 2018. Spectral bands: semiannual: 0.3-0.7 years; annual: 0.7-1.5 years; QBO: $1.5-3$ years.

between the QBO and the Madden-Julian oscillation (MJO) (e.g., Kuma 1990; Yoo and Son 2016; Hendon and Abhik 2018). Martin et al. (2019) found that static stability anomalies representing those associated with the QBO secondary circulation might help explain the reported relationship between the QBO and the MJO. Any connection between convection and the QBO should be impacted by buffer zone dynamics. Second, the secondary circulation of the QBO influences temperature and the distribution of radiatively active tracers (e.g., ozone, stratospheric water vapor) (Plumb and Bell 1982; Randel et al. 1998; Giorgetta and Bengtsson 1999; Fueglistaler and Haynes 2005). These tracers impact the stratospheric thermal structure and Earth's radiative balance.

One-dimensional models of the QBO, upon which much modern understanding of the phenomenon is based, are not well suited to study the buffer zone. These models implement a zero-wind lower boundary condition, and numerical simulations show that such models form a narrow buffer zone maintained by a balance between wave drag and vertical diffusion. Because these models do not spontaneously attenuate the QBO away from the lower boundary, the altitude of the lower boundary is typically chosen to match the climatological bottom of the QBO. Whereas in the real atmosphere, waves that drive the QBO might propagate several kilometers before reaching the QBO, the model waves initiated at the lower boundary reach the model QBO in several times less distance (details depend on model formulation, not discussed further). Saravanan (1990) proposed that the buffer zone might filter out waves with phase speeds close to zero, a dynamic that cannot be resolved in models with an unrealistically narrow buffer zone. Such models also cannot address questions such as: What sets the vertical extent of the QBO? Can the QBO propagate to the bottom of a vertically distributed wave source? Can the QBO impact the mean wind below the QBOactive region in order to modulate the waves that drive it?

Despite these model shortcomings, several idealized studies have examined the lower boundary of the QBO, reaching ambivalent conclusions about buffer zone dynamics. Plumb (1977) separated the wave source from the bottom of the model domain by setting the wave dissipation to zero near the bottom of the domain, but the wave source and lower extent of the QBO were still 
effectively collocated away from the lower boundary. Saravanan (1990) formed a buffer zone by imposing an exponentially decaying upwelling profile intended to represent the top of the Hadley cell. Although the model in Saravanan (1990) used a modified version of the Plumb (1977) parameterization of gravity wave drag, they based their qualitative argument for how the buffer zone formed on the Lindzen and Holton (1968) parameterization of gravity wave drag. The two parameterizations propagate information differently in the vertical, and arguments based on one parameterization do not necessarily apply to the other (Campbell et al. 2005). Held et al. (1993) found a QBO-like oscillation in $2 \mathrm{D}$ radiative-convective equilibrium that propagated down to the surface, a phenomenon that is further investigated in Nishimoto et al. (2016) and Bui et al. (2017). Dunkerton (1985) found that in a twodimensional simulation of the QBO, increasing the lateral momentum diffusion coefficient weakened the QBO, especially once the momentum decay time became smaller than the QBO period. Dunkerton (1997) used a 1D model to show that in the absence of horizontal advection by the Hadley cell in the troposphere, their simulated QBO propagated into the troposphere. They argued that in addition to the vertical mean advection of Saravanan (1990), horizontal mean advection by the Hadley cell might play a role in forming the buffer zone.

Only some modern general circulation models can spontaneously simulate a QBO (e.g., 5 of 47 CMIP5 model contributions (Schenzinger et al. 2017)). A common deficiency found in these simulations is that the QBO does not propagate far enough downward, vanishing an average of $10 \mathrm{hPa}$ higher up in the stratosphere in models than in observations (Schenzinger et al. 2017). The simulated QBO has been found to propagate farther down when the vertical resolution near the tropopause is increased (Anstey et al. 2016; Geller et al. 2016). But, interpreting the impact of model resolution or other processes on buffer zone formation is difficult without theoretical understanding of buffer zone dynamics.

With Saravanan (1990) implicating vertical mean advection and Dunkerton (1997) implicating both vertical and horizontal mean advection in the formation of the buffer zone, it seems plausible that several processes might be capable of producing a buffer zone. Which properties do these attenuating processes share? If several processes could independently attenuate the QBO, then which attenuating process does Earth's QBO encounter first? To answer these questions, we examine the angular momentum budget of the buffer zone in two meteorological reanalyses.
In section 2, we introduce the meteorological reanalyses we will be using: MERRA-2 and ERA-Interim. In section 3, we consider two generic ways to form a buffer zone: 1) weak wave-driven acceleration and/or 2) cancellation of the wave-driven acceleration. We show that weak wave-driven acceleration is insufficient to explain the low QBO power in the buffer zone. We present cross-spectral diagnostics on the phase shift between the wave-driven acceleration and the wind, which imply that the QBO is attenuated by damping. In section 4, we show that horizontal eddy and mean momentum fluxes strongly damp relative angular momentum anomalies in the buffer zone. In section 5 , we interpret the well-known correlation between QBO winds and Earth's length of day as supporting a conceptual model of the buffer zone in which QBO angular momentum anomalies are mixed between the QBO and the far field, with some anomalous angular momentum ultimately reaching the solid Earth.

\section{Global reanalyses}

Information from meteorological analyses is necessary to study the angular momentum budget of the buffer zone region because the only term in the budget that is directly observed is the net tendency. As meteorological analyses are weakly constrained in the buffer zone and there is the potential for compensating errors among terms in the budget, we perform our analysis using two systems: MERRA-2 and ERAInterim. Our primary results are presented using MERRA-2 because not all terms in ERA-Interim are available to us. Where possible, we use ERA-Interim to identify whether our results are robust between the two reanalyses.

We use the 3-hourly MERRA-2 assimilation product on pressure levels from January 1980 through November 2018 (GMAO 2015a,b; Gelaro et al. 2017). MERRA-2 publishes all terms required to close the momentum budget, including the model tendencies from gravity wave drag, turbulence, moist processes, and the analysis tendency. The QBO in MERRA-2 is primarily driven by the nonorographic gravity wave drag (Coy et al. 2016). The nonorographic gravity wave parameterization is from Garcia and Boville (1994), which follows Lindzen (1981). The background nonorographic gravity wave stress is imposed a priori at $400 \mathrm{hPa}$, then dissipates as a function of the wind state (Molod et al. 2015). Detailed aspects of the MERRA-2 momentum budget will be discussed later.

We use 6-hourly ERA-Interim on pressure levels (Dee et al. 2011; ECMWF 2011). The analysis tendency from ERA-Interim is not available to us. 


\section{Buffer zone formation}

\section{a. Definition}

Saravanan (1990) defined the QBO buffer zone as a "quiescent zone near the lower boundary [of the model]." Earth's buffer zone cannot be defined in terms of model boundaries, but a physically based interpretation of their definition is that the buffer zone is the region between the wave source and the lower extent of the QBO. We propose a general definition of the buffer zone as the region where the QBO would exist were it not for dynamical interference by processes unrelated to the QBO.

The bottom of the buffer zone is related to the source of the vertically propagating waves, which is assumed to be independent of the QBO. Vertically propagating waves relevant to driving the $\mathrm{QBO}$ are thought to originate from tropospheric deep convection (e.g., Baldwin and Dunkerton 2001). Deep convective circulations often extend up to $200 \mathrm{hPa}$, but rapidly become rare above $200 \mathrm{hPa}$, well below the active QBO (Fueglistaler et al. 2009).

The top of the buffer zone is identified as the level below which the zonal-mean zonal wind is no longer in a QBO regime. Determining that a region is outside the QBO regime requires a simplified model for how the power of zonal-mean zonal wind varies in a QBO regime. The simplest model would be that the QBO power is constant with height, in which case the top of the buffer zone would occur once the QBO power has decayed to some fraction of its maximum. But, for a given wave momentum flux divergence, the resulting acceleration is inversely proportional to density, which adds vertical structure to the QBO power. The simplest model that accounts for "density scaling" is to assume that QBO power is inversely proportional to density. [Density scaling neglects possible saturation of the QBO power (e.g., Plumb 1977), but this drawback should not limit its applicability to the lower levels of the QBO region.] The top of the buffer zone can be defined as the level at which the power of the zonal-mean zonal wind falls below some fraction of its density scaling.

\section{b. Vertical structure of $Q B O$ power}

Figure 2 shows the spectral power at $\mathrm{QBO}$ frequencies of the wind tendency and gravity wave drag, and their density scalings referenced to $30 \mathrm{hPa}$. Below $70 \mathrm{hPa}$, the power of the wind tendency decays rapidly below its density scaling, suggesting that any reasonable threshold value (e.g., $5 \%, 1 \%$ ) will identify the top of the buffer zone between 100 and $70 \mathrm{hPa}$.

The buffer zone can be formed by 1 ) weak wave-driven acceleration and/or 2) cancellation of the wave-driven acceleration. Considering limiting cases, the buffer zone

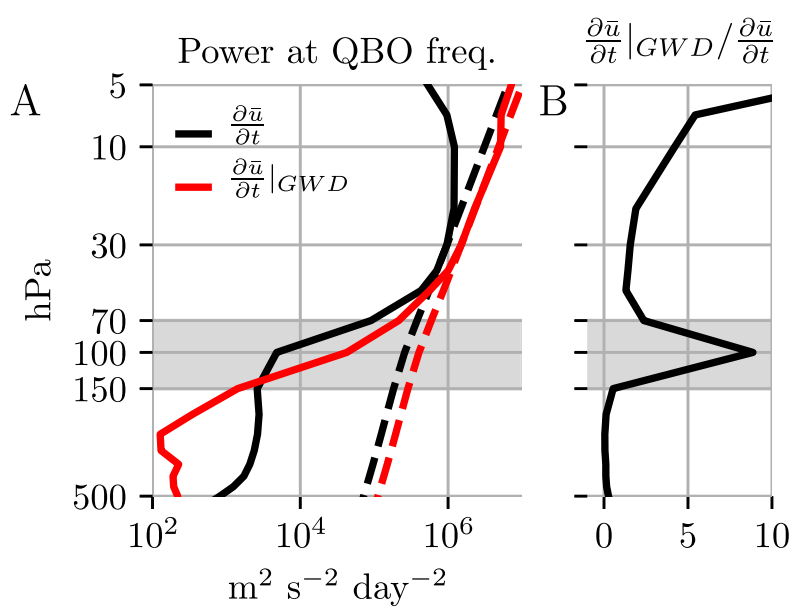

FIG. 2. Power of QBO wind tendency and gravity wave drag. (a) Spectral power at QBO periods (1.5-3 years) for the wind tendency (solid black) and gravity wave drag (solid red). Density scaling referenced to $30 \mathrm{hPa}$ for wind tendency (dashed black) and gravity wave drag (dashed red). (b) Ratio of two terms in (a): gravity wave drag divided by tendency. Data are from MERRA-2 averaged in longitude and from $10^{\circ} \mathrm{S}$ to $10^{\circ} \mathrm{N}$. The shaded gray region identifies the approximate buffer zone region (uncertainties in defining the buffer zone are discussed in sections $3 a$ and 6).

could have strong wave-driven acceleration and strong cancellation of the wave-driven acceleration, implicating cancellation in forming the buffer zone. Or, the buffer zone could have vanishing wave-driven acceleration, implicating weak wave-driven acceleration in forming the buffer zone. Figure 2 shows that the QBO power at $100 \mathrm{hPa}$ in MERRA-2 results from an intermediate case: the gravity wave drag is one order of magnitude below its density scaling (partially implicating weak wave-driven acceleration), and the wind tendency is one order magnitude below the gravity wave drag (partially implicating cancellation). The dynamics of weak wave-driven acceleration are beyond the scope of this paper, because wavedriven acceleration is both a cause and a consequence of the buffer zone (see the discussion section). This paper will focus on the dynamics of cancellation of wave-driven acceleration, which is necessary to explain the low QBO power in the buffer zone.

\section{c. Cancellation of wave-driven acceleration}

In the buffer zone, the power of the gravity wave drag exceeds that of the net wind tendency (Fig. 2), indicating that other forces cancel some of the gravity wave drag. We define two types of cancellation: opposition and damping. Opposition is exactly out of phase with the wave-driven acceleration; damping is exactly out of phase with the wind. Opposition occurs when the wavedriven acceleration and cancellation of the wave-driven acceleration are both mediated by the same process. 

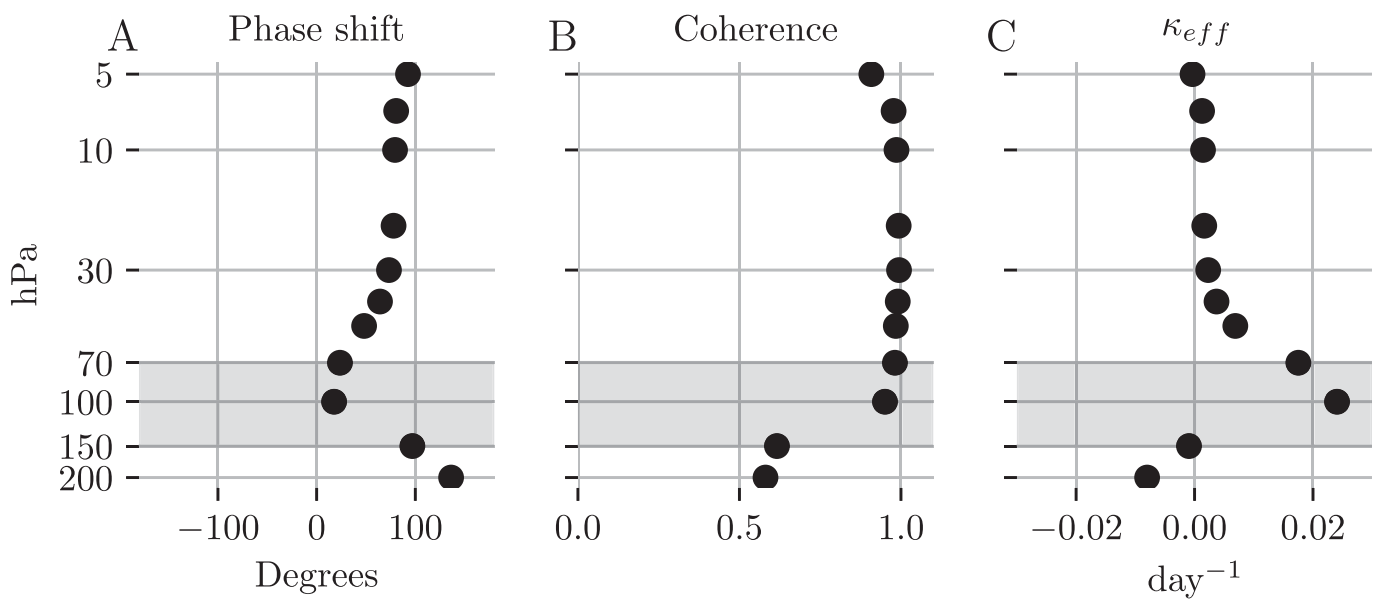

FIG. 3. Cross-spectral diagnostics between the gravity wave drag and wind indicate there is damping in the buffer zone. (a) Phase shift and (b) coherence between gravity wave drag and zonal wind at QBO frequency. (c) Effective damping rate $\kappa_{\text {eff }}$ implied by phase shift based on Eq. (2). Data are from MERRA-2 averaged in longitude and from $10^{\circ} \mathrm{S}$ to $10^{\circ} \mathrm{N}$. From the full cross-spectrum, only the QBO period band of 820 days is shown. The shaded gray region identifies the approximate buffer zone region (uncertainties in defining the buffer zone are discussed in sections $3 \mathrm{a}$ and 6 ).

Damping occurs when the wave-driven acceleration forms an angular momentum anomaly, which decays in time through relaxational angular momentum exchange with the far field. (Here, we refer to the far field as any part of the atmosphere outside the active QBO or buffer zone in the horizontal or vertical.) Damping depends on the local value of the wind, but opposition need not. The following forced and damped system distinguishes opposition from damping:

$$
\frac{d \bar{u}}{d t}=(F-O) \sin (\omega t)-\kappa \bar{u} .
$$

The variables are wind $\bar{u}$, time $t$, wave-driven acceleration with amplitude $F$ at angular frequency $\omega$, opposition $O$, and damping $\kappa$. The phase shift in radians between the wave-driven acceleration and wind is as follows:

$$
\theta=\frac{\pi}{2}-\tan ^{-1}\left(\frac{\kappa}{\omega}\right)
$$

Without opposition or damping, the wave-driven acceleration and wind are in quadrature. Saravanan (1990) argued that the buffer zone is formed where vertical mean advection opposes the wave-driven acceleration. If opposition (not damping) formed the buffer zone, then the wave-driven acceleration and wind would remain in quadrature. Dunkerton (1997) added that horizontal mean advection by the Hadley cell prevents the QBO from propagating into the troposphere, presumably by damping the QBO. If damping formed the buffer zone, then the wave-driven acceleration and wind should become more in phase. We examine the phase shift between wave-driven acceleration and wind in MERRA-2.

\section{d. Evidence for damping in MERRA-2}

Using cross-spectral diagnostics at QBO frequencies, we calculate the phase shift between the gravity wave drag and the wind to determine if there is damping in the buffer zone.

Figure 3 shows that at and above $30 \mathrm{hPa}$, the phase shift between the gravity wave drag and the wind is close to $90^{\circ}$, implying negligibly small damping. Below $30 \mathrm{hPa}$, the phase shift approaches $0^{\circ}$, implying strong damping. The coherence drops significantly from 70 to $100 \mathrm{hPa}$, indicating that gravity wave drag ceases to be the main process that drives variability in the wind.

Strong damping is consistent with the argument of damping by horizontal mean advection from Dunkerton (1997) and does not support the argument of opposition by vertical mean advection. But, the presence of strong damping does not do any of the following: 1) rule out any possible role for opposition, 2) imply that the horizontal mean advection implicated in Dunkerton (1997) is responsible for the damping, 3) imply that the vertical mean advection implicated as an opposing term in Saravanan (1990) does not instead play a damping role, or 4) imply that other terms, such as eddy momentum fluxes, do not play a damping role. Therefore, in the next section, we diagnose the damping by each individual term in the momentum budget. 


\section{What terms cause the damping?}

Acknowledging that multiple terms might be independently capable of producing strong damping that forms a buffer zone, and lacking a strong theoretical prior for which terms actually do so in nature, we use MERRA-2 to diagnose damping terms in the buffer zone and implicate the strongest damping terms in the formation of the buffer zone.

\section{a. Momentum equation}

We analyze the angular momentum equation to diagnose which terms are responsible for damping the QBO. Total atmospheric angular momentum per unit mass $m$ can be decomposed into Earth $\left(m_{\Omega}\right)$ plus relative $\left(m_{r}\right)$ angular momentum as follows:

$$
m=\underbrace{\Omega a^{2} \cos ^{2} \phi}_{m_{\Omega}}+\underbrace{u a \cos \phi}_{m_{r}} \text {. (3) }
$$

The atmosphere has been assumed to be shallow so that distance from the center of the Earth is approximated as Earth radius $a$. The material derivative of total angular momentum equation is

$$
\frac{d m}{d t}=-a \cos \phi\left(\frac{1}{a \cos \phi} \frac{\partial \Phi}{\partial \lambda}+g \frac{\partial \tau_{\lambda}}{\partial p}\right),
$$

where $(t, p, \phi, \lambda)$ is (time, pressure, latitude, longitude), $\Phi$ is the geopotential, and $\tau_{\lambda}$ is the zonal stress component. Zonal-mean angular momentum is materially conserved in the absence of zonal stress. Computing the zonal mean of Eq. (4), assuming continuity, leads to the flux form of Eq. (4):

$$
\begin{gathered}
\frac{\partial \overline{m_{r}}}{\partial t}+\frac{1}{a \cos \phi} \frac{\partial \bar{v} \bar{m}_{r} \cos \phi}{\partial \phi}+\frac{1}{a \cos \phi} \frac{\partial \overline{v^{\prime} m_{r}^{\prime}} \cos \phi}{\partial \phi} \\
-f \bar{v} a \cos \phi+\frac{\partial}{\partial p}\left(\bar{\omega} \bar{m}_{r}\right)+\frac{\partial}{\partial p}\left(\overline{\omega^{\prime} m_{r}^{\prime}}\right)=X .
\end{gathered}
$$

where overbars indicate zonal means and primes deviations therefrom. The terms on the left-hand side, in order, will be referred to as net tendency, horizontal mean momentum flux divergence, horizontal eddy momentum flux divergence, Coriolis torque, vertical mean momentum flux divergence, and vertical eddy momentum flux divergence. The term $f$ is the Coriolis parameter, $X$ represents unresolved tendencies, which in the MERRA-2 momentum budget are gravity wave drag, moist processes, turbulence, and the analysis tendency. Equation (5) can be integrated with a mass element $a \cos \phi d \lambda a d \phi d p / g$ to produce a budget of the mass-weighted relative angular momentum in the QBO domain. Angular momentum integrated in the $\mathrm{QBO}$ domain varies in time, indicating that the QBO exchanges angular momentum with the far field.

\section{b. Momentum budget in reanalyses}

Figure 4 shows terms in the budget of deseasonalized relative angular momentum density in log-pressure coordinates composited with respect to QBO phase. Deseasonalized anomalies in relative angular momentum density in log-pressure coordinates are contoured. When a positive tendency from a given term coincides with the transition from negative to positive relative angular momentum, such a term helps drive the QBO. When a negative tendency from a given term coincides with the transition from negative to positive relative angular momentum, such a term works against the QBO. For example, Fig. 4 shows that gravity wave drag helps drive the QBO, and horizontal eddy momentum flux divergence works against the QBO in the buffer zone. The momentum fluxes between 200 and $100 \mathrm{hPa}$ are large and not always coherent with the QBO. Figure S1 in the online supplemental material shows a duplicate of Fig. 4, but with hatching blocking out regions where the signal is below the noise. Notably, the large horizontal eddy momentum flux divergence in the lowermost stratosphere mostly exceeds the noise, and plays an important role in working against the QBO. To a lesser degree, the vertical mean momentum flux divergence also works against the QBO there. The MERRA-2 tendencies from turbulence and moist processes are not shown, as they are small compared to the other terms in the stratosphere (notably, their power at QBO frequencies in the stratosphere is at least two orders of magnitude lower than the power of the gravity wave drag).

Figure 4 shows that the net tendency in the buffer zone has smaller magnitude than several individual terms in the budget. In fact, the spectral power of the net tendency at QBO frequencies in the buffer zone is lower than that of all other terms shown in Fig. 4. Because the net tendency is the constrained sum of several large terms, there is a risk that errors in individual terms will lead to compensating errors in other terms that are as large as the net tendency.

If MERRA-2 does not simulate observed QBO tendencies through either resolved or parameterized momentum fluxes, then the net tendency will be produced by the analysis tendency. Figure $4 \mathrm{~g}$ shows that in MERRA-2, the analysis tendency is generally small, though it might weakly reinforce angular momentum anomalies in the lower stratosphere, for example, by providing easterly tendencies within easterly angular momentum anomalies, most evidently around $70 \mathrm{hPa}$. 

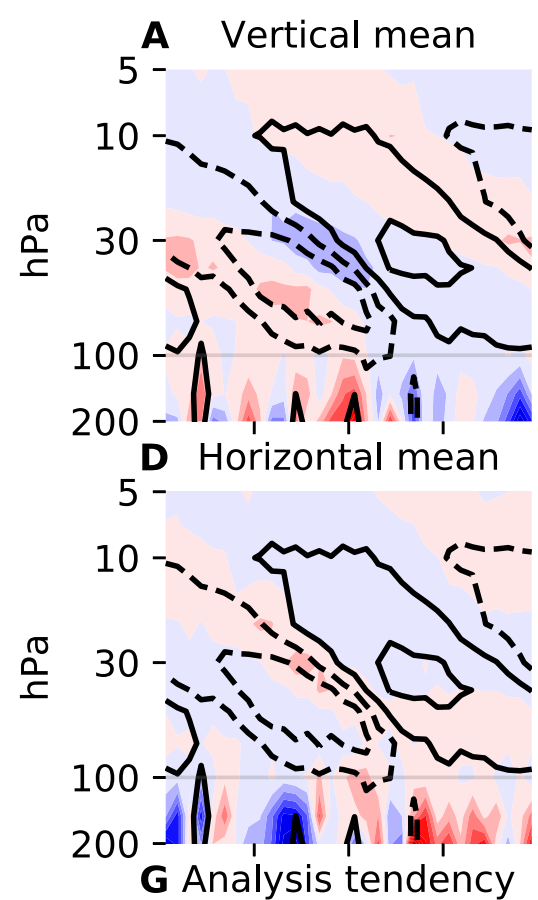
5 -

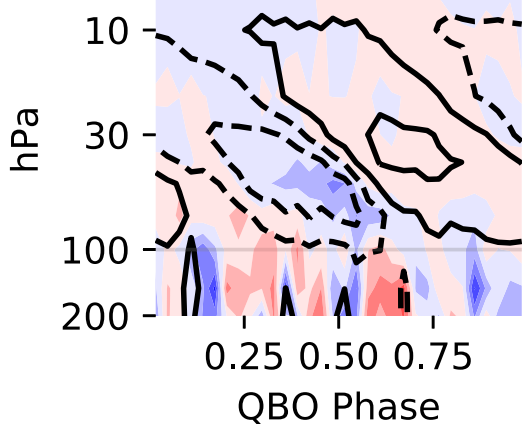

B Vertical eddy

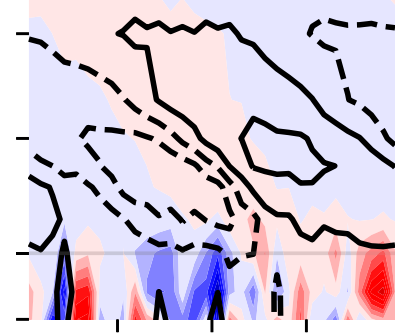

E Horizontal eddy

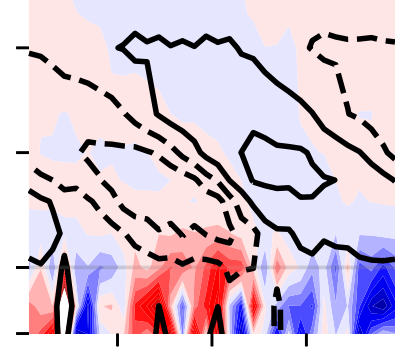

H Net tendency

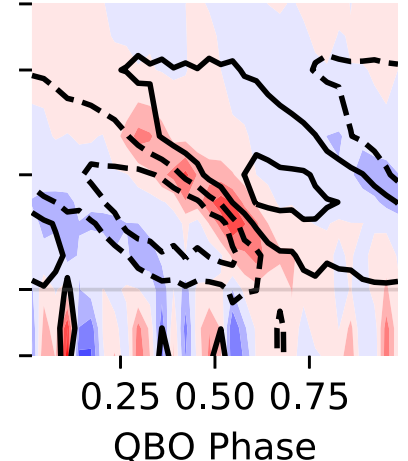

C Gravity wave drag
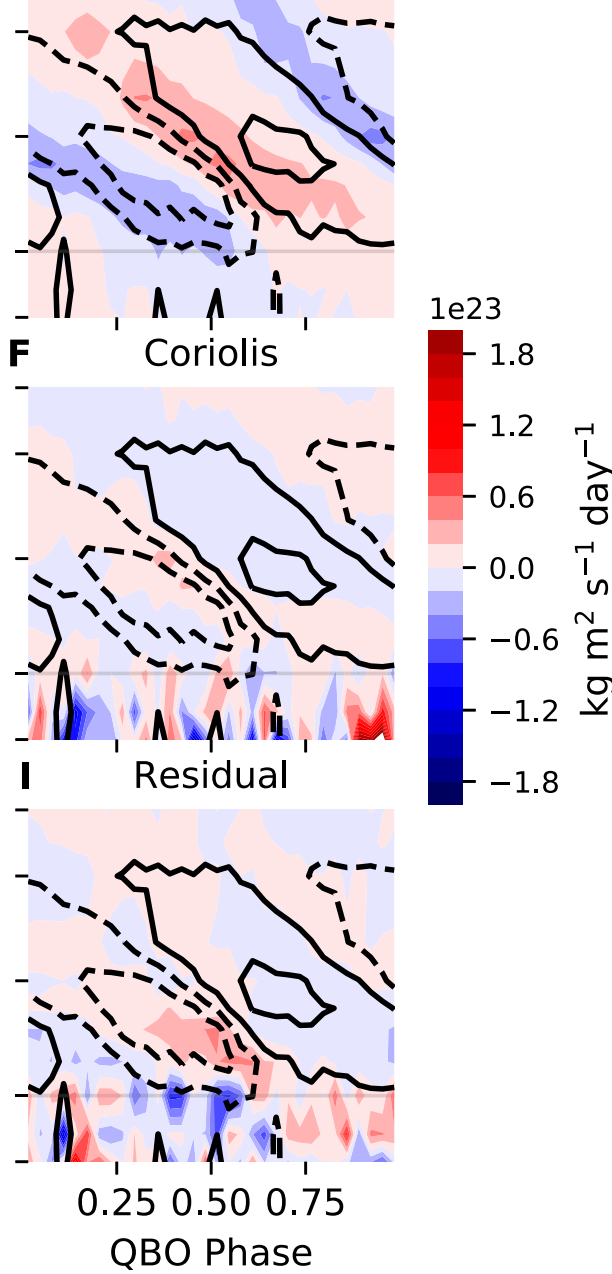

FIG. 4. Composite of relative angular momentum tendencies with respect to QBO phase. QBO phase is calculated from the principal components of the two leading empirical orthogonal functions of equatorial zonal wind from 70 to $10 \mathrm{hPa}$ following Wallace et al. (1993), and is composited across 32 bins. Shading indicates the time tendencies due to significant terms in relative angular momentum budget. Contours are relative angular momentum contoured at $[-9,-3,3,9] \times 10^{23} \mathrm{~kg} \mathrm{~m}^{2} \mathrm{~s}^{-1}$ (dashed lines are negative values). Data are MERRA-2 averaged monthly, deseasonalized, integrated from $10^{\circ} \mathrm{S}$ to $10^{\circ} \mathrm{N}$, and multiplied by log-pressure density (i.e., pressure/gravitational acceleration).

Figure $4 \mathrm{c}$ shows that most of the QBO driving in MERRA-2 comes from the gravity wave drag, with some help from the vertical eddy momentum flux divergence in Fig. 4b. Coy et al. (2016) argued that the QBO in MERRA-2 was driven more by physically based terms, a potential improvement over the QBO in the older MERRA, which was driven strongly by the analysis tendency.

Resolving the QBO tendencies with physically based terms instead of the analysis tendency should indicate improvement in MERRA-2, although it might hide other deficiencies. Vertical eddy momentum flux divergence is conspicuously small as a driver of the QBO in
MERRA-2 compared to in ERA-Interim. Dunkerton (1997) argued that $25 \%-50 \%$ of the QBO wave driving came from global-scale equatorial waves, which should be explicitly resolved in the reanalyzed vertical eddy momentum flux divergence. Kelvin waves are important global-scale waves that could help drive the easterly-to-westerly QBO transition. Analyzing High Resolution Dynamics Limb Sounder data, Alexander and Ortland (2010) found that Kelvin waves contributed about half of the wave forcing to descending westerly shear zones. Ern and Preusse (2009) found that Kelvin waves in ERA-Interim are responsible for $15 \%-30 \%$ of the total wave driving in 


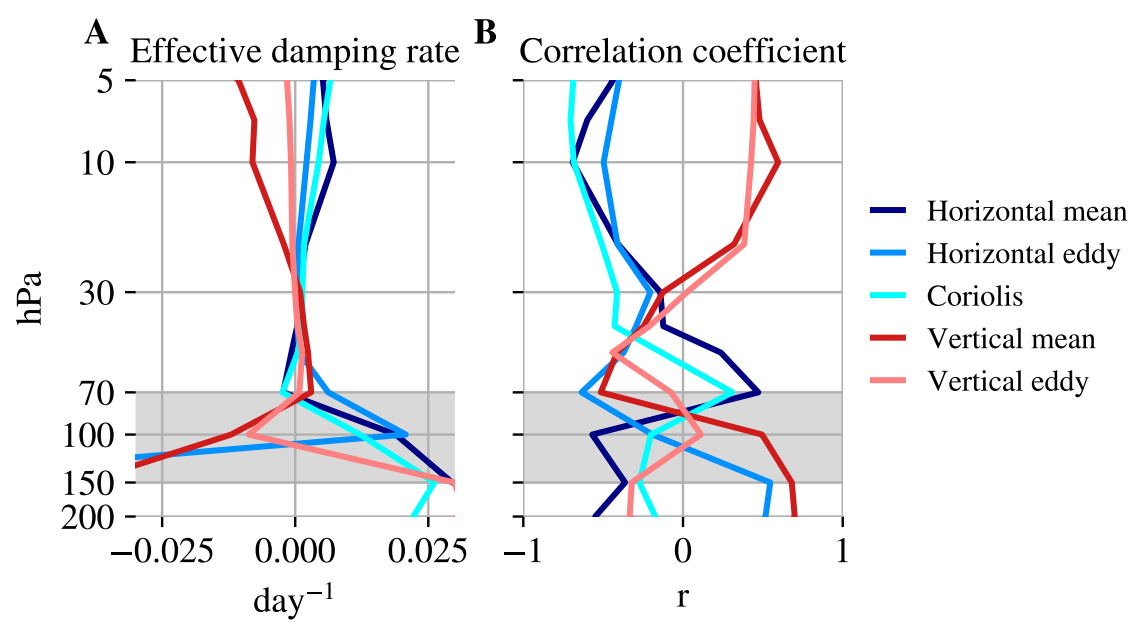

FIG. 5. Effective damping rates for major terms in the relative angular momentum budget. (a) Effective damping rates computed as the negative reciprocal of the least squares regression slope of the tendency vs deseasonalized relative angular momentum. (b) Correlation coefficients associated with the linear regression. Data are from MERRA-2 averaged monthly, in longitude, and from $10^{\circ} \mathrm{S}$ to $10^{\circ} \mathrm{N}$. The shaded gray region identifies the approximate buffer zone region (uncertainties in defining the buffer zone discussed in sections $3 \mathrm{a}$ and 6 ).

the stratosphere from $10^{\circ} \mathrm{S}$ to $10^{\circ} \mathrm{N}$, on the low end of the estimate from Dunkerton (1997). Flannaghan and Fueglistaler (2013) found that ERA-Interim contains discrete Kelvin waves that propagate from the troposphere into the stratosphere. This Kelvin wave activity increased after ERA-Interim began to assimilate temperatures from Constellation Observing System for Meteorology, Ionosphere, and Climate (COSMIC). Thus, with MERRA-2 having less wave activity than ERA-Interim, which in turn likely underestimates wave activity, it is plausible to assume that MERRA-2 underestimates wave activity.

Even by conservative estimates of the global-scale QBO wave driving, MERRA-2 appears deficient in its resolved wave driving of the QBO. Perhaps the increased background nonorographic gravity wave stress in MERRA-2, which shifted the net tendencies from being driven by the analysis tendency in MERRA to being driven by the gravity wave drag in MERRA-2, partially compensates for deficiencies in resolving the vertically propagating tropical waves that drive the QBO (e.g., Kelvin waves, mixed Rossbygravity waves). The reliance of the MERRA-2 QBO on parameterized waves is consistent with Holt et al. (2016), who showed that in a free-running simulation using a GCM similar to that used in MERRA-2, the QBO was strongly driven by parameterized waves even at high spatial resolution. Given the lack of robustness between models on what drives the QBO, caution should be exercised when interpreting angular momentum budgets in the QBO region and buffer zone.

\section{c. Effective damping rates}

We compute effective damping rates to find which terms form the buffer zone in MERRA-2 and ERAInterim. The effective damping rate is the negative slope of the least squares regression line between the tendency and the relative angular momentum. A positive effective damping rate means that when relative angular momentum is anomalously high, the tendency due to that given term tends to be negative. The least squares approach is predicated on the assumption that QBO angular momentum anomalies in the buffer zone interact with the far field in the same way that other angular momentum anomalies do. Such an approach permits us to evaluate how the atmosphere would respond to the presence of a QBO in regions where the QBO is presently attenuated.

Figure 5 shows the damping rates estimated at each level in the QBO region in MERRA-2. In Fig. 5a, horizontal mean momentum flux divergence and horizontal eddy momentum flux divergence are responsible for the strongest damping at $100 \mathrm{hPa}$, each with a damping time scale of approximately 50 days. The Coriolis torque at $100 \mathrm{hPa}$ has an effective damping time scale of 82 days. These damping time scales are much shorter than the QBO period and are expected to efficiently mute the QBO. Figure 5b shows that horizontal mean momentum flux divergence has a higher correlation coefficient 

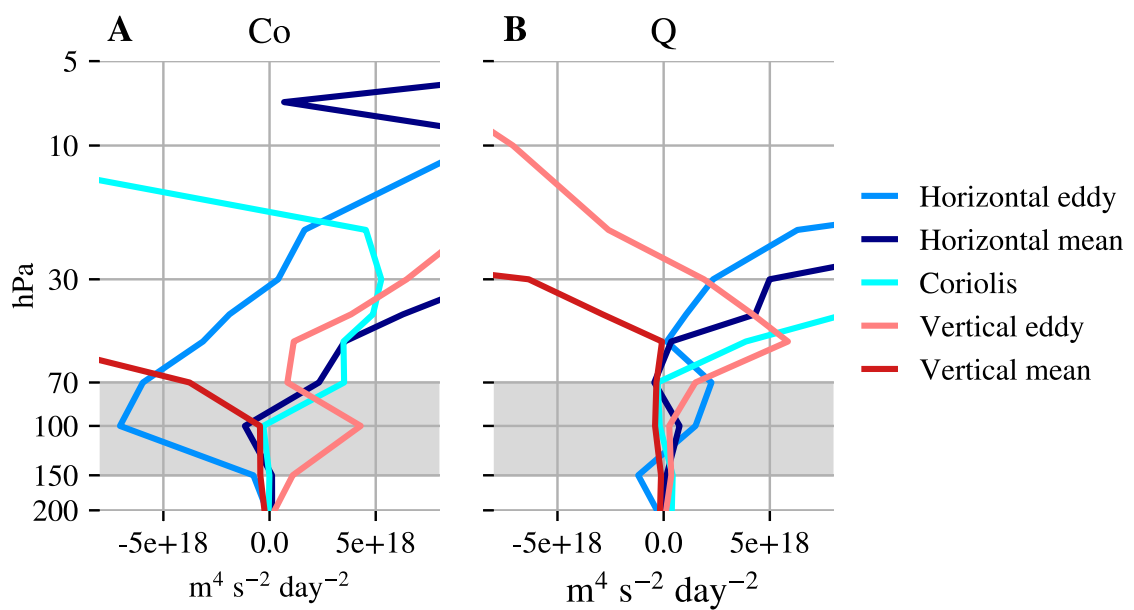

FIG. 6. (a) Cospectrum and (b) quadrature spectrum between the labeled terms and the gravity wave drag. All terms are from the MERRA-2 deseasonalized relative angular momentum budget averaged from $10^{\circ} \mathrm{S}$ to $10^{\circ} \mathrm{N}$ and are averaged over QBO frequencies (1.5-3 years). The shaded gray region identifies the approximate buffer zone region (uncertainties in defining the buffer zone are discussed in sections $3 \mathrm{a}$ and 6).

$(r=-0.57)$ than horizontal eddy momentum flux divergence $(r=-0.19)$ at $100 \mathrm{hPa}$. Whereas horizontal eddy momentum flux divergence only damps strongly at $100 \mathrm{hPa}$, horizontal mean momentum flux divergence and Coriolis torque damp strongly at and below $100 \mathrm{hPa}$. In ERA-Interim, horizontal mean momentum flux divergence is responsible for the strongest damping at $100 \mathrm{hPa}$, with a damping time scale of approximately 30 days $(r=-0.64)$ (Fig. S3).

Thus, neither reanalysis implicates vertical mean momentum flux divergence in forming the buffer zone, as in the mechanism of Saravanan (1990). Both reanalyses implicate horizontal mean momentum flux divergence, as argued in Dunkerton (1997), with ERA-Interim implicating horizontal mean momentum flux divergence almost exclusively and MERRA2 equally implicating horizontal mean momentum flux divergence and horizontal eddy momentum flux divergence at $100 \mathrm{hPa}$. Strong damping by the horizontal mean momentum flux divergence and Coriolis torque extends down into the troposphere, supporting the argument from Dunkerton (1997) that horizontal mean interactions with the Hadley cell would destroy any QBO signal in the upper troposphere.

\section{d. Cancellation at $Q B O$ frequencies}

The previous analysis of damping rates combined information from all possible frequencies, which is appropriate if the damping behavior is frequency independent. We now use cross-spectral diagnostics to analyze cancellation of gravity wave drag specifically at QBO frequencies. Figure 6 shows cross-spectral diagnostics between selected terms in the relative angular momentum budget and the gravity wave drag. Negative values of the cospectrum indicate out-of-phase behavior. Exact opposition presents as a negative value of the cospectrum and zero quadrature spectrum. In the weak damping limit, damping presents as a negative value of the quadrature spectrum, but in the strong damping limit, damping presents as a negative value of the cospectrum. In the intermediate damping case, damping presents as negative values of both the quadrature spectrum and cospectrum.

Figure 6 shows that vertical mean momentum flux divergence produces strong damping down to $70 \mathrm{hPa}$, consistent with arguments that upwelling weakens the QBO (e.g., Saravanan 1990; McIntyre 1994). But, the damping by vertical mean momentum flux divergence decays away strongly within the buffer zone, suggesting that it might not form the buffer zone, consistent with the least squares results in Fig. 5. Horizontal eddy momentum flux divergence produces strong damping at both 70 and $100 \mathrm{hPa}$. Vertical eddy momentum flux divergence generally reinforces the gravity wave drag, which would be expected if MERRA-2 was resolving some amount of global-scale wave driving of the QBO. Horizontal mean momentum flux divergence reinforces the gravity wave drag in the active QBO region, but cancels the gravity wave drag at $100 \mathrm{hPa}$. The Coriolis torque reinforces the gravity wave drag at QBO frequencies in the buffer zone despite producing a positive frequency-independent effective damping rate.

Because the effective damping rates in section $4 \mathrm{c}$ were computed using all angular momentum anomalies, they 
indicate the damping that the QBO would experience if it penetrated farther downward. In contrast, the crossspectral diagnostics in Fig. 6 only apply where the gravity wave drag is strong enough to drive QBO variability. Therefore, the small magnitude of the cospectrum in the upper troposphere does not imply the absence of terms that could attenuate the QBO.

\section{e. Using physical priors to distinguish opposition from damping}

We have shown that angular momentum tendencies cancel some of the gravity wave drag (Fig. 2), and that phase shifts between the wave-driven acceleration and wind imply there must be damping in the buffer zone (Fig. 3). However, we have not definitively ruled out a role for opposition. Particularly, once a region is in the strong damping limit, it can be difficult to disentangle whether there is also opposition.

Physical priors assist in distinguishing opposition from damping. Opposition can plausibly be caused by only vertical mean advection and no other terms. Therefore, if a term other than vertical mean advection produces cancellation, such a term is likely damping and not opposing. Vertical mean advection can oppose gravity wave drag if both processes are proportional to the vertical shear. In Lindzen and Holton (1968), gravity wave drag is parameterized based on a continuum of waves breaking at their critical layers, and the wave drag can be formulated as a downward advection, which is proportional to the vertical shear. Therefore, using the Lindzen and Holton (1968) parameterization could permit vertical mean advection to oppose gravity wave drag. In Plumb (1977), gravity wave drag is parameterized based on constant wave dissipation, and the gravity wave drag at a given level does not depend on the vertical shear. Therefore, using the Plumb (1977) parameterization would not feature opposition of gravity wave drag by vertical mean advection. Parameterizing gravity wave drag remains challenging and several plausible parameterizations exist today with different behaviors. However, since we find that the strongest cancellation does not arise from the vertical mean momentum flux divergence (which contains the vertical mean advection), it is unlikely that the buffer zone is formed by opposition to gravity wave drag irrespective of the parameterization, and instead damping is responsible.

Because angular momentum is globally conserved, damping concerns the exchange of angular momentum between the QBO and the far field. If the exchange of angular momentum between the QBO and the far field mixes the two regions toward some intermediate value, then such exchange will appear as a damping of the QBO angular momentum anomalies.
The effective damping rate from horizontal mean advection should scale as follows, assuming that the far field momentum is much smaller than (or uncorrelated with) the QBO momentum:

$$
-\bar{v} \frac{\partial \overline{m_{r}}}{\partial y} \sim-\bar{v} \frac{\bar{m}_{r \mathrm{QBO}}-\bar{m}_{r \mathrm{far}}}{\Delta y} \sim-\bar{v} \frac{\bar{m}_{r \mathrm{QBO}}}{\Delta y} \sim-\kappa_{\bar{v}} \bar{m}_{r \mathrm{QBO}},
$$

where $\Delta y$ is the latitudinal scale of $1000 \mathrm{~km}$ (approximately the QBO half-width), and damping rate from mean advection $\kappa_{v}$ equals $\bar{v} / \Delta y$. A characteristic velocity scale of $1 \mathrm{~m} \mathrm{~s}^{-1}$ leads to a damping rate of $\sim 10$ days, on the order of the diagnosed damping rates in Fig. 5. Identical arguments apply to the vertical mean advection with $\bar{w} \sim 0.1 \mathrm{~mm} \mathrm{~s}^{-1}$ divided by $\Delta z \sim 1 \mathrm{~km}$ yielding $\kappa_{\bar{w}} \sim 100$ days. For vertical advection, the assumption that $\bar{m}_{r}$ ar is uncorrelated with the QBO does not apply in the interior of the QBO. Vertical mean advection should only act as a damping term near the bottom of the QBO region, as it advects the quiescent winds of the buffer zone upward into the active QBO region.

Horizontal eddies were shown to damp angular momentum anomalies in the buffer zone, especially on QBO time scales (Figs. 5 and 6). The mechanism for how horizontal eddies produce damping remains unclear. If transport of angular momentum by horizontal eddies was diffusive in the buffer zone, then horizontal eddies would damp angular momentum there. However, several factors suggests that angular momentum transports by atmospheric waves are nondiffusive: 1) atmospheric waves can transport angular momentum across long distances, 2) atmospheric waves can transport angular momentum upgradient, and 3) planetary waves only produce easterly torques where they break. Given these reasons that waves might not act diffusively, the mechanism for how horizontal eddies produce such large effective damping rates deserves future attention.

\section{Correlation of QBO winds with length of day}

Classical one-dimensional models of the QBO are forced by symmetric wave fluxes, so the waves do not add net angular momentum to the domain. Yet, the angular momentum of the model domain still oscillates in phase with the QBO. The net tendencies of angular momentum in the QBO domain are maintained by angular momentum fluxes through the lower boundary of the model, which represent exchange between the QBO and the far field. In Fig. 4, we showed that in analogous fashion to the 1D theory, Earth's buffer zone mediates the exchange of angular momentum between the QBO and the far field. 


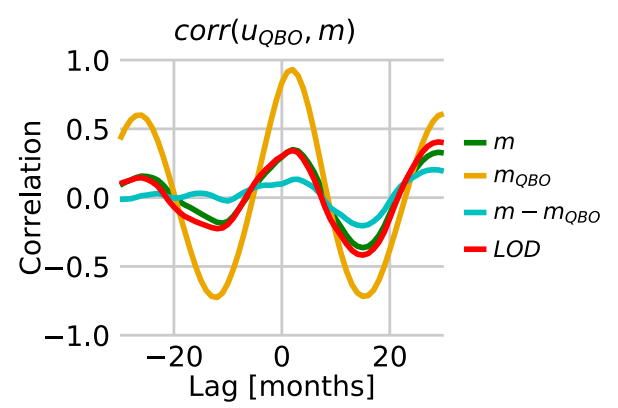

FIG. 7. Lag correlation between monthly deseasonalized variables related to the globally-integrated atmospheric angular momentum and $u_{\mathrm{QBO}}$, where $u_{\mathrm{QBO}}$ is the zonal wind at the equator and $30 \mathrm{hPa}$ in MERRA-2, $m$ is the globally integrated total angular momentum of the atmosphere in MERRA-2, $m_{\mathrm{QBO}}$ is the angular momentum integrated over $20^{\circ} \mathrm{S}-20^{\circ} \mathrm{N}$ and $10-100 \mathrm{hPa}$ in MERRA-2, and LOD is the length of day from COMB2017 (Ratcliff and Gross 2018) ftp://euler.jpl.nasa.gov/ keof/combinations/2017/comb2017_midnight.lod. LOD is inferred from Earth orientation measurements and is derived independently of atmospheric angular momentum. We compute the monthly average of the LOD and then remove the seven gravest Fourier modes as in Paek and Huang (2012). Positive shifts mean $u_{\mathrm{QBO}}$ leads the momentum variable.

What happens to the anomalous angular momentum once it reaches the far field? Anomalous angular momentum could remain in the atmosphere, in which case the QBO would represent a repartitioning of angular momentum between the QBO domain and the far field. In reality, some of the anomalous QBO angular momentum is transferred into the solid Earth, leading to a lagged correlation between the QBO winds and the length of day (LOD) (Chao 1989; Egger et al. 2007; Paek and Huang 2012).

Figure 7 shows the lag correlation between the zonal wind at the equator and $30 \mathrm{hPa}\left(u_{\mathrm{QBO}}\right)$ and four measures of angular momentum. The large correlation between $u_{\mathrm{QBO}}$ and $M_{\mathrm{QBO}}$ at zero lag indicates that $u_{\mathrm{QBO}}$ is a suitable proxy for $M_{\mathrm{QBO}}$. The large lag correlation between $u_{\mathrm{QBO}}$ and $M_{\mathrm{QBO}}$ at \pm 28 months highlights the regularity of the QBO. The large correlation between LOD and $u_{\mathrm{QBO}}$ indicates that some of the angular momentum transferred from the QBO domain to the far field is then transferred into the solid Earth by atmospheric torques. The standard deviation of the atmospheric angular momentum, which gives the characteristic magnitude of the repartitioning of angular momentum between the atmosphere and the solid Earth, is $2 \times 10^{25} \mathrm{~kg} \mathrm{~m}^{2} \mathrm{~s}^{-1}$. The standard deviation of relative angular momentum in the QBO region $\left(10^{\circ} \mathrm{S}-10^{\circ} \mathrm{N}, 100-10 \mathrm{hPa}\right)$ is $2.9 \times 10^{24} \mathrm{~kg} \mathrm{~m}^{2} \mathrm{~s}^{-1}$. Thus, the QBO region contains merely $1.5 \%$ of the total atmospheric mass, but accounts for almost $15 \%$ of the variability in global atmospheric relative angular momentum.
Figure 7 shows that in order to close the angular momentum budget of the QBO, it is necessary to consider angular momentum in the QBO region, the far field atmosphere, and the solid Earth. This paper has focused on the flows of momentum between the QBO region and the far field. We showed that much of the exchange of angular momentum between the QBO region and the far field occurs in the buffer zone, and that this exchange presents as a strong damping by horizontal terms in the angular momentum budget. Once in the far field, angular momentum anomalies can be mixed down to the surface, where they are transferred to the solid Earth via the friction torque or mountain torque. The pathways taken by the QBO angular momentum anomalies from the far field atmosphere to the solid Earth remain largely unexplored. Figure 8 summarizes our conceptual model that the buffer zone "ventilates" QBO angular momentum anomalies to the far field atmosphere, some of which eventually reach the solid Earth.

\section{Discussion}

Figure 2a showed that weak wave-driven acceleration contributes to the formation of the buffer zone, but is insufficient to fully explain the formation of the buffer zone. Weak wave-driven acceleration could cause low QBO power, but weak wave-driven acceleration could also be a consequence of low QBO power. In the QBO theory of Holton and Lindzen (1972), wave dissipation increases for more extreme values of the wind, so a decrease in the QBO power could cause a decrease in the power of the gravity wave drag, which could feed back to further decrease QBO power. This positive feedback would need to be started by an initial decrease in QBO power, which could be caused by the damping described in this paper. The strength of this positive feedback cannot be constrained by reanalysis alone, but deserves further attention.

Despite being a dynamically active tracer, angular momentum might fit into a paradigm of stratospheric transport applied to chemical tracers. The active QBO region coincides with the dynamically isolated region of the stratosphere known as the "tropical pipe", so named because air masses there tend to ascend with limited horizontal mixing (Plumb 1996). The dynamical isolation of the tropical pipe is exemplified by the stratospheric water vapor tape recorder, a pattern characterized by the advection of water vapor anomalies established at the cold-point tropopause, but can be observed in other tracers (Mote et al. 1996). Plausibly, the dynamical isolation that permits tracer anomalies to coherently propagate upward by advection also 


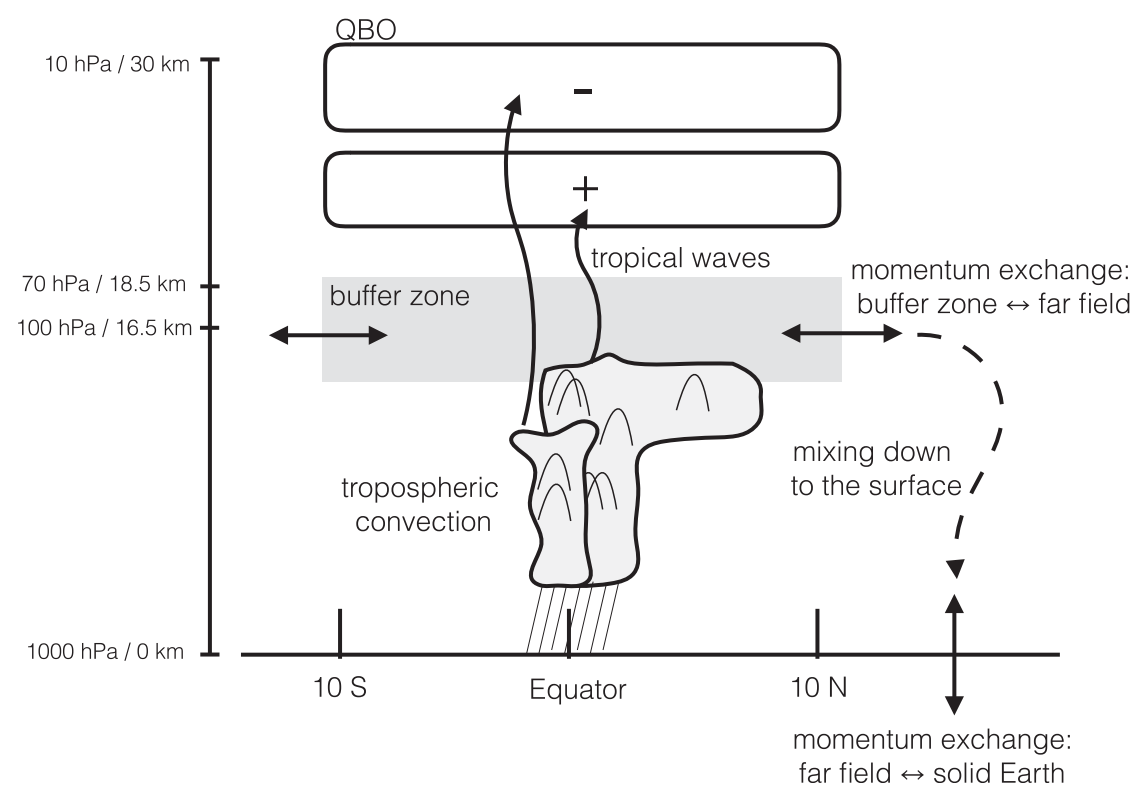

FIG. 8. Buffer zone schematic: QBO zonal-mean zonal wind on a typical day (contours), with plus signs and minus signs indicating westerly and easterly shear zones, respectively. The arrows indicate angular momentum exchange: tropical waves transport angular momentum upward into the QBO region, creating QBO anomalies. The QBO angular momentum anomalies propagate into the buffer zone, where these anomalies are transferred to the far field. Some of the far-field angular momentum is transferred into the solid Earth.

permits momentum anomalies to propagate downward by the wave-mean flow mechanism of Lindzen and Holton (1968), without experiencing "toxic" horizontal mixing (Dunkerton 1997). Below the tropical pipe is the tropical tropopause layer and the region we have identified as the buffer zone. In the buffer zone, which we have identified for its horizontal mixing of angular momentum that attenuates the QBO, other atmospheric tracers also experience rapid, quasi-isentropic transport (Randel et al. 2001; Fueglistaler et al. 2009).

We return to the question of defining the buffer zone. We define the buffer zone as the region in which the QBO would exist absent dynamical interference. A buffer zone definition would ideally be based on observables and be objectively applicable in varied contexts, for example, deep-time Earth, exoplanets, and laboratory experiments. The top and bottom of the buffer zone are defined by separate criteria, and both encode assumptions about QBO dynamics.

An ideal definition for the top of the buffer zone would identify where the QBO becomes weaker than expected from density scaling. This definition is based on observables (wind and density) and is objectively applicable in varied contexts. For a truly generic definition, the QBO period would have to be selected to match the relevant period, as a QBO in another context need not have a 28 -month period.
Identifying the bottom of the buffer zone cannot easily be achieved with observations alone. Assuming that the QBO can propagate to the bottom of a vertically deep wave source, absent dynamical interference, the bottom of the buffer zone corresponds to the bottom of the wave source. In the MERRA-2 world, the bottom of the buffer zone is $400 \mathrm{hPa}$, the source of the nonorographic gravity waves that drive the QBO. Yet, actual gravity waves do not originate at a single level nor do they necessarily originate so low in the atmosphere. Alexander and Vincent (2000) argue that the observed gravity wave spectra in the lower stratosphere are inconsistent with isotropic origins in the lower troposphere and more consistent with isotropic origins near the tropopause.

What about the latitudinal extent of the buffer zone? We consider the buffer zone to be mainly confined to the tropics, that is, spanning the latitudes over which the QBO exhibits large power in the stratosphere. However, defining the buffer zone as the region where the QBO would exist absent dynamical interference raises the question of whether the QBO could propagate meridionally given the current wave driving. There has long been a question of what role laterally propagating waves of midlatitude origin play in driving the QBO. Dunkerton (1983) showed that a 1D model forced by only one vertically propagating wave (instead of the 
typical two) and a crude parameterization of laterally propagating planetary waves produced a QBO. The recent QBO disruption of 2015/16 highlighted that laterally propagating Rossby waves can dissipate in the QBO region (Osprey et al. 2016; Newman et al. 2016; Lin et al. 2019). There is no evidence for lateral critical layer propagation in the observed QBO (Hamilton 1984), but it is not clear whether lateral critical layer propagation is forbidden by wave dynamics or whether potential laterally propagating signals are destroyed by dynamical interference. Dynamical interference, ideally independent of the QBO, could be difficult to establish for laterally propagating signals because the QBO might influence midlatitude dynamics by modulating the location of the zero-wind line (Holton and Tan 1980; O'Sullivan 1997).

\section{Conclusions}

The buffer zone is the region in which the QBO would exist absent dynamical interference. Rather than spontaneously producing a buffer zone, classical 1D QBO models enforce its existence through the zero-wind lower boundary condition.

Analyzing MERRA-2, we show that the power of the QBO at $100 \mathrm{hPa}$ is an order of magnitude less than the power of the gravity wave drag, implying cancellation of the gravity wave drag. The phase shift between the gravity wave drag and the wind indicates that the QBO is strongly damped below $50 \mathrm{hPa}$. From a least squares approach, the damping in the buffer zone region is primarily due to horizontal eddy momentum flux divergence, horizontal mean momentum flux divergence, and Coriolis torque in MERRA-2, and due to horizontal mean momentum flux divergence in ERA-Interim. From a frequency-dependent cross-spectral approach, the damping in the buffer zone at QBO frequencies is primarily due to horizontal eddy momentum fluxes. The buffer zone mediates the relaxational exchange of angular momentum between the QBO and the far field. Angular momentum exported from the QBO region can ultimately reach the solid Earth, leading to the observed correlation between QBO angular momentum and the length of day.

Acknowledgments. We thank Chiung-Yin Chang, Isaac Held, Pu Lin, Nathaniel Tarshish, and Yi Zhang for helpful discussions at various stages of this work. We also thank two anonymous reviewers for their helpful and constructive reviews. This work resulted in part from a collaboration with Valentina Aquila. This material is based upon work supported by the National Science Foundation Graduate Research Fellowship Program under
Grant DGE-1656466. Any opinions, findings, and conclusions or recommendations expressed in this material are those of the authors and do not necessarily reflect the views of the National Science Foundation.

\section{REFERENCES}

Alexander, M. J., and R. A. Vincent, 2000: Gravity waves in the tropical lower stratosphere: A model study of seasonal and interannual variability. J. Geophys. Res., 105, 17 983-17993, https://doi.org/10.1029/2000JD900197.

— tion Dynamics Limb Sounder (HIRDLS) data. J. Geophys. Res., 115, D24111, https://doi.org/10.1029/2010JD014782.

Anstey, J. A., J. F. Scinocca, M. Keller, J. A. Anstey, J. F. Scinocca, and M. Keller, 2016: Simulating the QBO in an atmospheric general circulation model: Sensitivity to resolved and parameterized forcing. J. Atmos. Sci., 73, 1649-1665, https://doi.org/ 10.1175/JAS-D-15-0099.1.

Baldwin, M. P., and T. J. Dunkerton, 2001: Stratospheric harbingers of anomalous weather regimes. Science, 294, 581-584, https://doi.org/10.1126/science.1063315.

Bui, H.-H., E. Nishimoto, S. Yoden, H.-H. Bui, E. Nishimoto, and S. Yoden, 2017: Downward influence of QBO-like oscillation on moist convection in a two-dimensional minimal model framework. J. Atmos. Sci., 74, 3635-3655, https://doi.org/10.1175/ JAS-D-17-0095.1.

Campbell, L. J., T. G. Shepherd, L. J. Campbell, and T. G. Shepherd, 2005: Constraints on wave drag parameterization schemes for simulating the quasi-biennial oscillation. Part I: Gravity wave forcing. J. Atmos. Sci., 62, 4178-4195, https:// doi.org/10.1175/JAS3616.1.

Chao, B. F., 1989: Length-of-day variations caused by El NiñoSouthern Oscillation and quasi-biennial oscillation. Science, 243, 923-925, https://doi.org/10.1126/science.243.4893.923.

Coy, L., K. Wargan, A.M. Molod, W.R. McCarty, and S. Pawson, 2016: Structure and dynamics of the quasi-biennial oscillation in MERRA-2. J. Climate, 29, 5339-5354, https://doi.org/ 10.1175/JCLI-D-15-0809.1.

Dee, D. P., and Coauthors, 2011: The ERA-Interim reanalysis: Configuration and performance of the data assimilation system. Quart. J. Roy. Meteor. Soc., 137, 553-597, https://doi.org/ 10.1002/qj.828.

Dunkerton, T. J., 1983: Laterally-propagating Rossby waves in the easterly acceleration phase of the quasi-biennial oscillation. Atmos.-Ocean, 21, 55-68, https://doi.org/10.1080/ 07055900.1983.9649155.

_ 1985: A two-dimensional model of the quasi-biennial oscillation. J. Atmos. Sci., 42, 1151-1160, https://doi.org/10.1175/ 1520-0469(1985)042<1151:ATDMOT>2.0.CO;2.

_ 1997: The role of gravity waves in the quasi-biennial oscillation. J. Geophys. Res., 102, 26053-26076, https://doi.org/10.1029/ 96JD02999.

Ebdon, R. A., and R. G. Veryard, 1961: Fluctuations in equatorial stratospheric winds. Nature, 189, 791-793, https://doi.org/10.1038/ $189791 \mathrm{a} 0$.

ECMWF, 2011: The ERA-Interim reanalysis dataset. Accessed 2 February 2013, https://www.ecmwf.int/en/forecasts/datasets/ archive-datasets/reanalysis-datasets/era-interim.

Egger, J., K. Weickmann, and K.-P. Hoinka, 2007: Angular momentum in the global atmospheric circulation. Rev. Geophys., 45, RG4007, https://doi.org/10.1029/2006RG000213. 
Ern, M., and P. Preusse, 2009: Quantification of the contribution of equatorial Kelvin waves to the QBO wind reversal in the stratosphere. Geophys. Res. Lett., 36, L21801, https://doi.org/ 10.1029/2009GL040493.

Flannaghan, T. J., and S. Fueglistaler, 2013: The importance of the tropical tropopause layer for equatorial Kelvin wave propagation. J. Geophys. Res. Atmos., 118, 5160-5175, https://doi.org/ 10.1002/jgrd.50418.

Fueglistaler, S., and P. H. Haynes, 2005: Control of interannual and longer-term variability of stratospheric water vapor. J. Geophys. Res., 110, D24108, https://doi.org/10.1029/2005JD006019.

— A. E. Dessler, T. J. Dunkerton, I. Folkins, Q. Fu, and P. W. Mote, 2009: Tropical tropopause layer. Rev. Geophys., 47, RG1004, https://doi.org/10.1029/2008RG000267.

Garcia, R. R., and B. A. Boville, 1994: "Downward control" of the mean meridional circulation and temperature distribution of the polar winter stratosphere. J. Atmos. Sci., 51, 2238-2245, https:// doi.org/10.1175/1520-0469(1994)051<2238:COTMMC >2.0.CO;2.

Gelaro, R., and Coauthors, 2017: The Modern-Era Retrospective Analysis for Research and Applications, Version 2 (MERRA-2). J. Climate, 30, 5419-5454, https://doi.org/10.1175/ JCLI-D-16-0758.1.

Geller, M. A., and Coauthors, 2016: Modeling the QBOImprovements resulting from higher-model vertical resolution. J. Adv. Model. Earth Syst., 8, 1092-1105, https://doi.org/ 10.1002/2016MS000699.

Giorgetta, M. A., and L. Bengtsson, 1999: Potential role of the quasi-biennial oscillation in the stratosphere-troposphere exchange as found in water vapor in general circulation model experiments. J. Geophys. Res., 104, 6003-6019, https://doi.org/ 10.1029/1998JD200112.

GMAO, 2015a: MERRA-2 inst3_3d_asm_Np: 3d, 3-Hourly, Instantaneous, Pressure-Level, Assimilation, Assimilated Meteorological Fields V5.12.14. GES DISC, accessed 27 January 2019, https://doi.org/10.5067/QBZ6MG944HW0.

— 2015 b: MERRA-2 tavg3_3d_udt_Np: 3d, 3-Hourly, TimeAveraged, Pressure-Level, Assimilation, Wind Tendencies V5.12.4. GES DISC, accessed 27 January 2019, https://doi.org/ 10.5067/CWV0G3PPPWFW.

Hamilton, K., 1984: Mean wind evolution through the quasi-biennial cycle in the tropical lower stratosphere. J. Atmos. Sci., 41, 2113-2125, https://doi.org/10.1175/1520-0469(1984)041<2113: MWETTQ $>2.0 . \mathrm{CO} ; 2$.

Held, I. M., R. S. Hemler, and V. Ramaswamy, 1993: Radiativeconvective equilibrium with explicit two-dimensional moist convection. J. Atmos. Sci., 50, 3909-3927, https://doi.org/10.1175/ 1520-0469(1993)050<3909:RCEWET>2.0.CO;2.

Hendon, H. H., and S. Abhik, 2018: Differences in vertical structure of the Madden-Julian oscillation associated with the quasi-biennial oscillation. Geophys. Res. Lett., 45, 4419-4428, https://doi.org/10.1029/2018GL077207.

Holt, L. A., M. J. Alexander, L. Coy, A. Molod, W. Putman, and S. Pawson, 2016: Tropical waves and the quasi-biennial oscillation in a 7-km global climate simulation. J. Atmos. Sci., 73, 3771-3783, https://doi.org/10.1175/JAS-D-15-0350.1.

Holton, J. R., and R. S. Lindzen, 1972: An updated theory for the quasi-biennial cycle of the tropical stratosphere. J. Atmos. Sci., 29, 1076-1080, https://doi.org/10.1175/1520-0469(1972)029<1076: AUTFTQ > 2.0.CO;2.

, and H.-C. Tan, 1980: The Influence of the equatorial quasibiennial oscillation on the global circulation at $50 \mathrm{mb}$. J. Atmos. Sci., 37, 2200-2208, https://doi.org/10.1175/1520-0469(1980) 037<2200:TIOTEQ > 2.0.CO;2.
Kuma, K.-I., 1990: A quasi-biennial oscillation in the intensity of the intra-seasonal oscillation. Int. J. Climatol., 10, 263-278, https:// doi.org/10.1002/joc.3370100304.

Lin, P., I. Held, and Y. Ming, 2019: The early development of the 2015/2016 Quasi-Biennial Oscillation disruption. J. Atmos. Sci., 76, 821-836, https://doi.org/10.1175/JAS-D-18-0292.1.

Lindzen, R. S., 1981: Turbulence and stress owing to gravity wave and tidal breakdown. J. Geophys. Res., 86, 9707-9714, https:// doi.org/10.1029/JC086iC10p09707.

_ , and J. R. Holton, 1968: A theory of the quasi-biennial oscillation. J. Atmos. Sci., 25, 1095-1107, https://doi.org/10.1175/ 1520-0469(1968)025<1095:ATOTQB > 2.0.CO;2.

Martin, Z., S. Wang, J. Nie, and A. Sobel, 2019: The impact of the QBO on MJO convection in cloud-resolving simulations. J. Atmos. Sci., 76, 669-688, https://doi.org/10.1175/JAS-D-18-0179.1.

McIntyre, M. E., 1994: The quasi-biennial oscillation (QBO): Some points about the terrestrial QBO and the possibility of related phenomena in the solar interior. The Solar Engine and Its Influence on Terrestrial Atmosphere and Climate, E. Nesme-Ribes, Ed., Springer, 293-320, https://doi.org/10.1007/ 978-3-642-79257-1_18.

Molod, A., L. Takacs, M. Suarez, and J. Bacmeister, 2015: Development of the GEOS-5 atmospheric general circulation model: evolution from MERRA to MERRA2. Geosci. Model Dev., 8, 1339-1356, https://doi.org/10.5194/gmd-8-1339-2015.

Mote, P. W., and Coauthors, 1996: An atmospheric tape recorder: The imprint of tropical tropopause temperatures on stratospheric water vapor. J. Geophys. Res., 101, 3989-4006, https:// doi.org/10.1029/95JD03422.

Newman, P. A., L. Coy, S. Pawson, and L. R. Lait, 2016: The anomalous change in the QBO in 2015-2016. Geophys. Res. Lett., 43, 8791-8797, https://doi.org/10.1002/2016GL070373.

Nishimoto, E., S. Yoden, H.-H. Bui, E. Nishimoto, S. Yoden, and H.-H. Bui, 2016: Vertical momentum transports associated with moist convection and gravity waves in a minimal model of QBO-like oscillation. J. Atmos. Sci., 73, 2935-2957, https:// doi.org/10.1175/JAS-D-15-0265.1.

Osprey, S. M., N. Butchart, J. R. Knight, A. A. Scaife, K. Hamilton, J. A. Anstey, V. Schenzinger, and C. Zhang, 2016: An unexpected disruption of the atmospheric quasi-biennial oscillation. Science, 353, 1424-1427, https://doi.org/10.1126/science.aah4156.

O'Sullivan, D., 1997: Interaction of extratropical Rossby waves with westerly quasi-biennial oscillation winds. J. Geophys. Res., 102, 19461-19469, https://doi.org/10.1029/97JD01524.

Paek, H., and H.-P. Huang, 2012: A comparison of the interannual variability in atmospheric angular momentum and length-ofday using multiple reanalysis data sets. J. Geophys. Res., 117, D20102, https://doi.org/10.1029/2012JD018105.

Plumb, R. A., 1977: The interaction of two internal waves with the mean flow: Implications for the theory of the quasi-biennial oscillation. J. Atmos. Sci., 34, 1847-1858, https://doi.org/ 10.1175/1520-0469(1977)034<1847:TIOTIW>2.0.CO;2. _, 1996: A "tropical pipe" model of stratospheric transport. J. Geophys. Res., 101, 3957-3972, https://doi.org/10.1029/ 95JD03002.

_ , and R. C. Bell, 1982: A model of the quasi-biennial oscillation on an equatorial beta-plane. Quart. J. Roy. Meteor. Soc., $\mathbf{1 0 8}$, 335-352, https://doi.org/10.1002/qj.49710845604.

Randel, W. J., F. Wu, J. M. Russell, A. Roche, and J. W. Waters, 1998: Seasonal cycles and QBO variations in stratospheric $\mathrm{CH}_{4}$ and $\mathrm{H}_{2} \mathrm{O}$ observed in UARS HALOE data. J. Atmos. Sci., 55, 163-185, https://doi.org/10.1175/1520-0469(1998)055<0163: SCAQVI $>2.0 . \mathrm{CO} ; 2$. 
A. Gettelman, J. M. Russell, J. M. Zawodny, and S. J. Oltmans, 2001: Seasonal variation of water vapor in the lower stratosphere observed in Halogen Occultation Experiment data. J. Geophys. Res., 106, 14313-14325, https://doi.org/ 10.1029/2001JD900048.

Ratcliff, J., and R. Gross, 2018: Combinations of earth orientation measurements: SPACE2017, COMB2017, POLE2017. JPL Publ. 18-5, 25 pp., https://keof.jpl.nasa.gov/combinations/ SpaceCombPole_latest.pdf.

Reed, R. J., W. J. Campbell, L. A. Rasmussen, and D. G. Rogers, 1961: Evidence of a downward-propagating, annual wind reversal in the equatorial stratosphere. J. Geophys. Res., 66, 813-818, https://doi.org/10.1029/ JZ066i003p00813.

Saravanan, R., 1990: A multiwave model of the quasi-biennial oscillation. J. Atmos. Sci., 47, 2465-2474, https://doi.org/10.1175/ 1520-0469(1990)047<2465:AMMOTQ>2.0.CO;2.
Schenzinger, V., S. Osprey, L. Gray, and N. Butchart, 2017: Defining metrics of the quasi-biennial oscillation in global climate models. Geosci. Model Dev., 10, 2157-2168, https:// doi.org/10.5194/gmd-10-2157-2017.

Scinocca, J. F., 2003: An accurate spectral nonorographic gravity wave drag parameterization for general circulation models. J. Atmos. Sci., 60, 667-682, https://doi.org/10.1175/15200469(2003)060<0667:AASNGW>2.0.CO;2.

Wallace, J. M., R. L. Panetta, J. Estberg, J. M. Wallace, R. L. Panetta, and J. Estberg, 1993: Representation of the equatorial stratospheric quasi-biennial oscillation in EOF phase space. J. Atmos. Sci., 50, 1751-1762, https://doi.org/10.1175/1520-0469(1993) 050<1751:ROTESQ $>2.0$. CO; 2 .

Yoo, C., and S.-W. Son, 2016: Modulation of the boreal wintertime Madden-Julian oscillation by the stratospheric quasi-biennial oscillation. Geophys. Res. Lett., 43, 1392-1398, https://doi.org/ 10.1002/2016GL067762. 